\title{
The constitutive equations of finite strain poroelasticity in the light of a micro-macro approach
}

\author{
Patrick de Buhan ${ }^{a *}$, Xavier Chateau ${ }^{b}$, Luc Dormieux ${ }^{a}$ \\ acole Nationale des Ponts et Chaussées, CERMMO, 6-8 avenue Blaise Pascal, 77420 Champs-sur-Marne, France \\ b Laboratoire des Matériaux et des Structures du Génie Civil (UMR 113 CNRS-LCPC), 2 allée Kepler, 77420 Champs-sur-Marne, France
}

(Received 7 April 1997; revised and accepted 28 April 1998)

\begin{abstract}
After recalling the constitutive equations of finite strain poroelasticity formulated at the macroscopic level, we adopt a microscopic point of view which consists of describing the fluid-saturated porous medium at a space scale on which the fluid and solid phases are geometrically distinct. The constitutive equations of poroelasticity are recovered from the analysis conducted on a representative elementary volume of porous material open to fluid mass exchange. The procedure relies upon the solution of a boundary value problem defined on the solid domain of the representative volume undergoing large elastic strains. The macroscopic potential, computed as the integral of the free energy density over the solid domain, is shown to depend on the macroscopic deformation gradient and the porous space volume as relevant variables. The corresponding stress-type variables obtained through the differentiation of this potential turn out to be the macroscopic Boussinesq stress tensor and the pore pressure. Furthermore, such a procedure makes it possible to establish the necessary and sufficient conditions to ensure the validity of an 'effective stress' formulation of the constitutive equations of finite strain poroelasticity. Such conditions are notably satisfied in the important case of an incompressible solid matrix. (C) Elsevier, Paris
\end{abstract}

constitutive equation / finite strain poroelasticity / macroscopic approach / microscopic approach / incompressible solid matrix

\section{Introduction}

The so-called Biot approach to the constitutive behaviour of porous media is purely macroscopic. In the case of a poroelastic medium, it is based on the hypothesis that there exists a thermodynamic potential which is the free energy of the open porous system. As far as infinitesimal transformations are concerned, Biot's poroelasticity theory has been substantiated in a theoretical manner by means of homogenization techniques. One could refer for instance to the study by Auriault and Sanchez-Palencia (1977) where a periodic model is adopted and the use of asymptotic expansion techniques is advocated; or to Thompson and Willis (1991) and Cheng (1997), who succeeded in deriving the material coefficients of poroelasticity from micromechanical considerations.

A growing interest has been recently devoted to the use of poroelasticity at large strains, particularly in the field of biomechanics (Kowalczyk and Kleiber, 1994; van Campen et al., 1994). In a recent contribution, Cieszko and Kubik (1996) have developed a "macro-continuum" model of a fluid-saturated porous solid subject to large elastic deformations. Their analysis is based upon a description of the saturated material as an immiscible solid-fluid mixture, producing constitutive equations identical with those previously obtained by Biot (1972). However, no clear reference is made in this work to the microscopic scale of description, so that the form of the thermodynamic elastic potential has to be directly postulated at the macroscopic scale.

The present paper proposes a micro-macro approach which extends the justification of Biot's poroelasticity theory to the domain of large strains. It is based on the previous works of Hill (1971) or Ogden (1974) concerning

\footnotetext{
* Correspondence and reprints
} 
the overall response of inhomogeneous elastic solids under finite strain. They proved in particular that applying appropriate boundary conditions to a representative elementary volume of such a solid makes it possible to derive a macroscopic elastic constitutive law of the same kind as that postulated for the solid at the microscopic scale.

Before extending such a procedure to the case of fluid-saturated porous materials, it first appears necessary to briefly recall the basic features of the macroscopic poroelasticity theory at large strains. For more details, the interested reader can refer to the pioneering work of Biot $(1972,1977)$, recently reformulated by Coussy (1989, 1995). It should be emphasized that in the following, the term "skeleton" will be devoted to the description of the solid at the macroscopic scale, whereas the term "solid" or "solid matrix" refers to the microscopic scale. For the sake of simplicity, the analysis will be restricted to the situation of a porous medium saturated by a single fluid undergoing isothermal quasi-static evolutions. The motions considered are sufficiently slow that shear stresses due to viscosity are neglected throughout the fluid, whose action on the solid matrix is purely mechanical in the form of a homogeneous pressure distribution.

\section{Formulation of the poroelasticity theory: the macroscopic approach}

Let $d \Omega_{o}$ be an elementary domain of porous medium in its initial configuration and $d \Omega_{t}$ the domain occupied at time $t$ by the skeleton particle initially located in $d \Omega_{o}$. Referring to the microscopic scale, we also denote by $d \Omega_{\alpha}^{s}$ and by $d \Omega_{\alpha}^{f}$ with $\alpha=o, t$ the subdomains actually filled by the solid and the fluid respectively, so that $d \Omega_{\alpha}=d \Omega_{\alpha}^{s} \cup d \Omega_{\alpha}^{f}$.

By definition, $\Psi\left|d \Omega_{o}\right|$ denotes the free energy of both the fluid and solid materials contained in $d \Omega_{t}$. The increase in fluid mass in $d \Omega_{t}$ with respect to $d \Omega_{o}$ is denoted by $m\left|d \Omega_{o}\right|$, which defines the density $m$.

We denote by $\underline{\underline{\sigma}}$ the Cauchy total stress tensor. Let $\underline{\underline{F}}$ be the deformation gradient of the skeleton transformation and $\triangleq=\frac{1}{2}\left({ }^{T} \underline{\underline{F}} \cdot \underline{\underline{F}}-\underline{\underline{1}}\right)$ the Green-Lagrange strain tensor $\left({ }^{T} \underline{\underline{F}}\right.$ denotes the transpose of $\left.\underline{\underline{F}}\right)$. It can be shown that

$$
m=\rho^{f} J \phi-\rho_{o}^{f} \phi_{o}
$$

where $J=\operatorname{det} \underline{\underline{F}}$ is the volume strain $\left|d \Omega_{t}\right| /\left|d \Omega_{o}\right|, \rho^{f}$ the fluid density and $\phi$ the fluid volume fraction in the current configuration (porosity). The subscript "o" refers to the initial configuration. It is convenient to introduce the Piola-Kirchhoff stress tensor $\underline{\underline{\pi}}$ and the Boussinesq stress tensor $\underline{\underline{\theta}}$ respectively related to the Cauchy stress by

$$
\underline{\underline{\pi}}=J \underline{\underline{F}}^{-1} \cdot \underline{\underline{\sigma}} \cdot{ }^{T} \underline{\underline{F}}^{-1} ; \underline{\underline{\theta}}=J \underline{\underline{\sigma}} \cdot{ }^{T} \underline{\underline{F}}^{-1}
$$

and which satisfy

$$
\underline{\underline{\pi}}: \underline{\underline{\Delta}}=\underline{\underline{\theta}}:{ }^{T} \underline{\underline{\underline{F}}}
$$

With these notations, the general expression of the intrinsic dissipation $D$ associated with the skeleton behaviour (per unit volume in the initial configuration) is (Coussy, 1989)

$$
D=\underline{\underline{\pi}}: \underline{\underline{\Delta}}+g_{m} \dot{m}-\dot{\Psi}=\underline{\underline{\theta}}:{ }^{T} \underline{\underline{\dot{F}}}+g_{m} \dot{m}-\dot{\Psi} \geq 0
$$

where $g_{m}$ denotes the free enthalpy of the unit fluid mass. The particular case of poroelasticity is defined by two hypotheses, namely that $\Psi$ only depends on two state variables which are the strain tensor $\triangleq$ and the increase in fluid mass $m$, and that the intrinsic dissipation is equal to zero. Both assumptions combined with (4) lead to the constitutive equations of the open poroelastic system under the form

$$
\underline{\underline{\pi}}=\frac{\partial \Psi}{\partial \triangleq}(\triangleq, m), g_{m}=\frac{\partial \Psi}{\partial m}(\triangleq, m)
$$


Alternatively, if we consider $\Psi$ as a function of $\underline{\underline{F}}$ and $m$, we obtain

$$
\underline{\underline{\theta}}=\frac{\partial \Psi}{\partial \underline{\underline{F}}}(\underline{\underline{F}}, m), g_{m}=\frac{\partial \Psi}{\partial m}(\underline{\underline{F}}, m)
$$

The thermodynamic approach thus shows that the thermodynamic force associated with the increase in fluid mass $m$ is the free enthalpy $g_{m}$ and not the fluid pressure $p$. However, the fluid pressure proves to be more familiar and, for practical purposes, more convenient than the free enthalpy. Actually, Dormieux and Stolz (1992) and Dangla and Coussy (1996) have shown that the fluid pressure is the thermodynamic force associated with the "Lagrangian" porosity $J \phi$, i.e. referring again to the microscopic scale, the ratio $\left|d \Omega_{t}^{f}\right| /\left|d \Omega_{o}\right|$ between the volume $\left|d \Omega_{t}^{f}\right|$ filled in $d \Omega_{t}$ by the fluid and the total volume $\left|d \Omega_{o}\right|$ in the initial configuration of the skeleton. More precisely, let $\Psi^{s}$ be the density of free energy of the skeleton, which is related to $\Psi$ by

$$
\Psi^{s}=\Psi(\underline{\underline{\Delta}}, m)-\left(m+\rho_{o}^{f} \phi_{o}\right) \varphi_{m}
$$

where $\varphi_{m}$ is the free energy of the fluid unit mass (i.e. $\varphi_{m}=g_{m}-p / \rho^{f}$ ). We obtain

$$
g_{m} \dot{m}-\dot{\Psi}=p \frac{\dot{m}}{\rho^{f}}-\left(m+\rho_{o}^{f} \phi_{o}\right) \dot{\varphi}_{m}-\dot{\Psi}^{s}
$$

Recalling that $\dot{\varphi}_{m}=-p \overline{\left.\frac{\dot{1}}{\rho^{J}}\right)}$ and using (1), the above equation yields

$$
g_{m} \dot{m}-\dot{\Psi}=p \dot{\overline{J \phi}}-\dot{\Psi}^{s}
$$

The intrinsic dissipation now takes the form

$$
D=\underline{\underline{\theta}}:{ }^{T} \underline{\underline{\dot{F}}}+p \dot{\overline{J \phi}}-\dot{\Psi}^{s}
$$

Provided that the state equation of the fluid be given, i.e. that the function $g_{m}=g_{m}(p)$ be specified, (5) allows to consider that $p, \rho^{f}=1 / \frac{d g_{m}}{d p}$ as well as $J \phi=\left(m+\rho_{o}^{f} \phi_{o}\right) / \rho^{f}$ are functions of $\triangleq$ and $m$. Using the fact that $D$ is zero in (10), one finally derives a new formulation of the constitutive equations under the form

$$
\underline{\underline{\theta}}=\frac{\partial \Psi^{s}}{\partial \underline{\underline{F}}}(\underline{\underline{F}}, J \phi), p=\frac{\partial \Psi^{s}}{\partial(J \phi)}(\underline{\underline{F}}, J \phi)
$$

For a given state equation of the fluid, the different forms (5), (6) and (11) of the constitutive equations of the porous medium are equivalent.

\section{The micro-macro approach}

The constitutive equations of the porous medium have been derived in the previous section within the framework of a thermodynamic reasoning performed at the macroscopic scale, where the skeleton and fluid particles contained in an elementary volume $d \Omega_{t}$ are geometrically coincident (figure la). The analysis will now be conducted at the microscopic scale according to which $d \Omega_{t}$ is regarded as a volume containing a solid matrix occupying a subdomain $d \Omega_{t}^{s}$ of $d \Omega_{t}$, while the remaining part $d \Omega_{t}^{f}$ which forms the connected porous space is filled by a fluid (figure $l b$ ). Such a volume, called the representative elementary volume, is selected in such a way that it is sufficiently large with respect for instance to the characteristic pore size, while remaining 


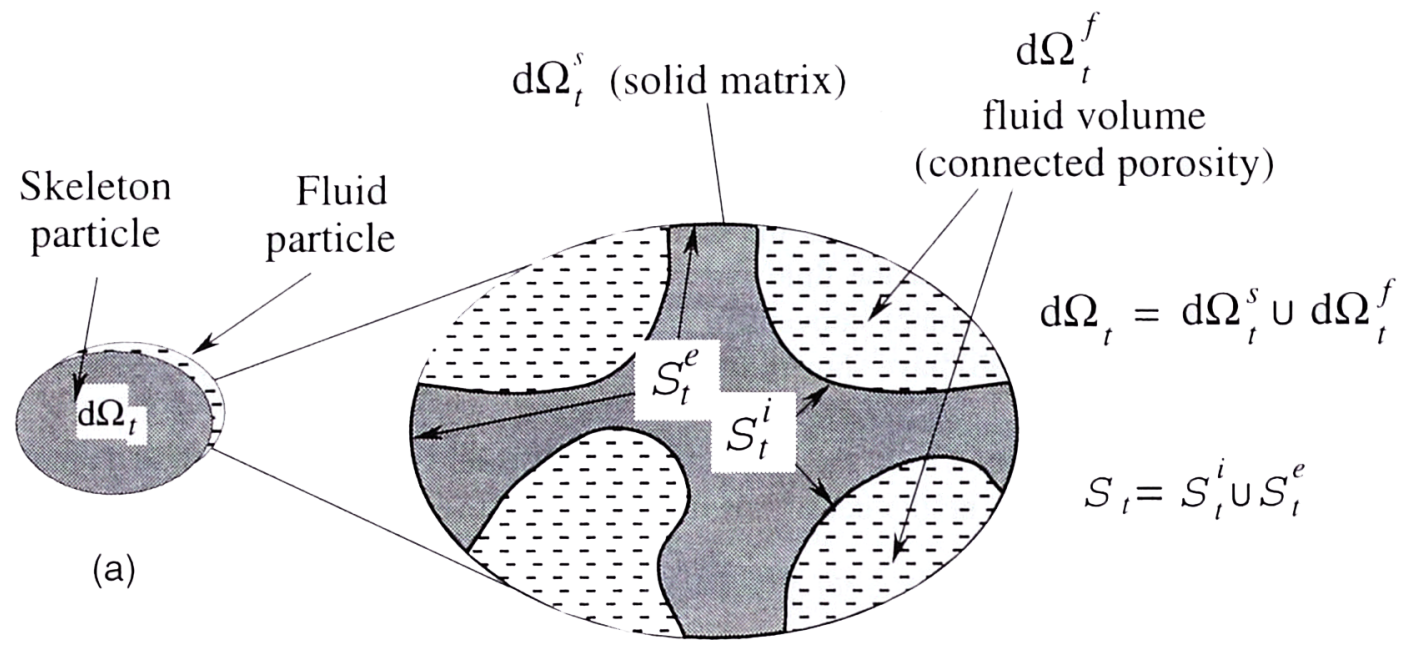

(b)

Figure 1. Perception of a satured porous medium at the macroscopic (a) and microscopic (b) scales in the current configuration at time $t$.

small enough when compared with the overall dimensions of the structure under consideration. Let $\mathcal{S}_{t}$ denote the boundary surface of the solid domain, so that $\mathcal{S}_{t}^{e}=\mathcal{S}_{t} \cap \partial\left(d \Omega_{t}\right)$ represents the external part of $\mathcal{S}_{t}$ and $\mathcal{S}_{t}^{i}$ the internal part of $\mathcal{S}_{t}$ which remains in contact with the fluid contained in $d \Omega_{t}$. Analogous notations where " $t$ " is replaced by " $O$ " are adopted for the solid in its initial configuration.

Assuming that the solid behaves elastically, the aim of the present section is to establish the constitutive equations of finite strain poroelasticity by means of a micro-macro approach. As in the macroscopic approach, the porous medium will be considered as an open system with a variable fluid mass. The representative elementary domain $d \Omega_{0}$ in the initial configuration of the porous medium must therefore be subjected to a loading characterized by either the change in fluid mass $m$ or the "Lagrangian" porosity $J \phi$, and by the tensor $\underline{\underline{F}}$ which represents the macroscopic deformation gradient of $d \Omega_{o}$ in the transformation of the skeleton. Accordingly, the boundary of $d \Omega_{t}$ is defined as the image of the boundary of $d \Omega_{o}$ by the homogeneous transformation associated with $\underline{\underline{F}}$ (figure 2).

The response of the solid part of $d \Omega_{0}$ to this loading is characterized by the function $\underline{x}=\underline{\Phi}^{s}(\underline{X})$, where $\underline{X}$ (resp. $\underline{x}$ ) denotes the position vector of a solid particle at the microscopic scale in the initial (resp. deformed) configuration. The tensor $\underline{\underline{F}}^{s}=\underline{\underline{\nabla}} \mathrm{X}^{\underline{\Phi}}$ is the deformation gradient at the microscopic scale and $\tilde{J}^{s}=\operatorname{det} \underline{\underline{\tilde{F}}}^{s}$ is the corresponding local volume strain. Denoting by $\tilde{\psi}$ the density of free energy of the solid, by $\underline{\underline{\tilde{\sigma}}}$ the Cauchy stress tensor and by $\underline{\underline{\theta}}=\tilde{J}^{s} \underline{\underline{\tilde{\sigma}}} \cdot\left({ }^{T} \underline{\underline{\underline{F}}}\right)^{-1}$ the Boussinesq stress tensor in the solid at the microscopic scale, the state equation of the solid writes

$$
\underline{\underline{\theta}}=\frac{\partial \tilde{\psi}}{\partial \underline{\underline{F}}^{s}}\left(\underline{\underline{F}}^{s}\right)
$$

According to the physical meaning of $\underline{\underline{F}}$ introduced as the macroscopic deformation gradient of the skeleton, the loading to which the solid is subjected is defined by the following boundary conditions

$$
\text { on } \mathcal{S}_{o}^{e}: \underline{\Phi}(\underline{X})=\underline{\underline{F}} \cdot \underline{X} ; \text { on } \mathcal{S}_{o}^{i}: \underline{\underline{\theta}} \cdot \underline{N}=-p \tilde{J}^{s} \underline{\underline{\tilde{F}}}^{s}-1 \cdot \underline{N}
$$



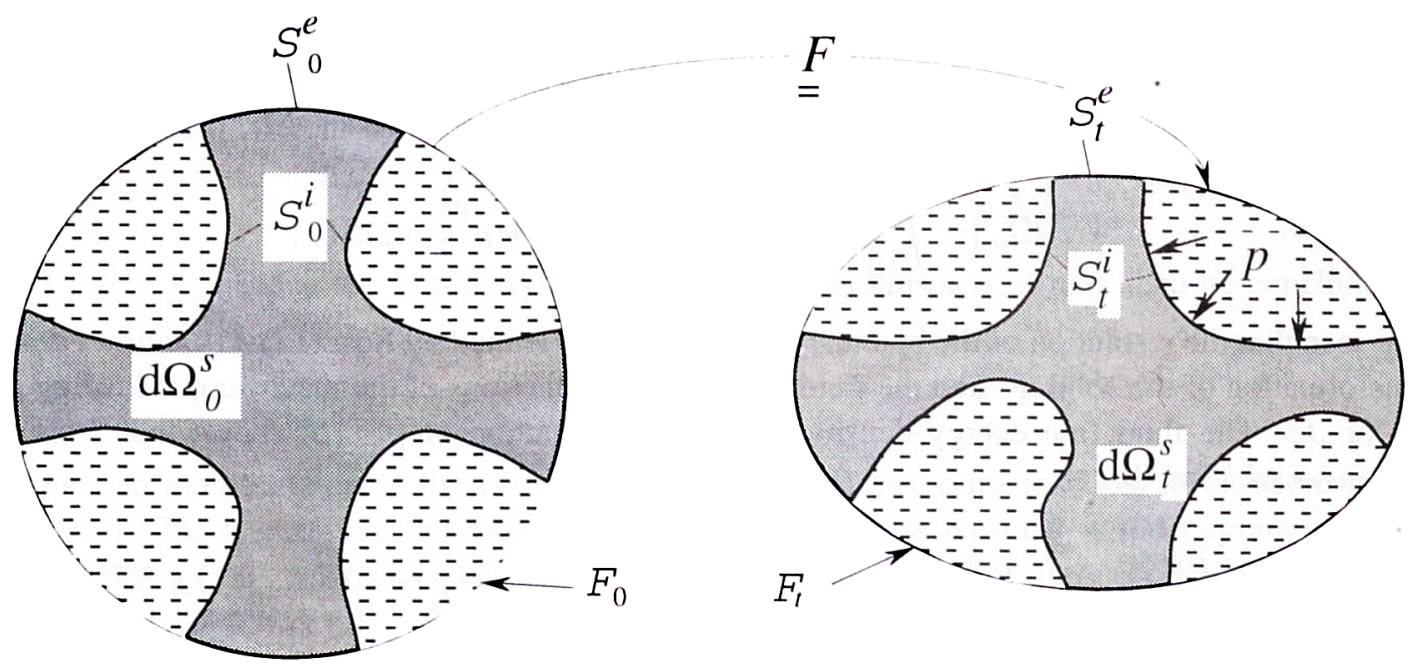

Figure 2. Initial and current configurations of a representative elementary volume of saturated porous medium (microscopic scale).

where $\underline{N}$ denotes the outer unit normal vector in the initial configuration of the solid and $p$ is the fluid pressure. It is worth noting that the above equations correspond to "mixed" boundary conditions, in the sense that the displacement is prescribed on $\mathcal{S}_{o}^{e}$, whereas the condition relating to $\mathcal{S}_{o}^{i}$ expresses, in terms of Lagrangian quantities, the fact that the fluid is exerting a uniform pressure on $\mathcal{S}_{t}^{i}$. In addition, the Boussinesq and Cauchy stress fields must comply with the equilibrium conditions with no body force which can be written either on the initial configuration or on the deformed one

$$
\text { on } d \Omega_{o}^{s} \operatorname{Div} \underline{\underline{\tilde{\theta}}}=0 ; \text { on } d \Omega_{t}^{s} \operatorname{div} \underline{\underline{\tilde{\sigma}}}=0
$$

where Div (resp. div) denotes the divergence operator on the initial (resp. current) configuration.

The set of Eqs (12), (13) and (14) defines a finite strain elastic boundary value problem on the solid part of the representation volume. It will be assumed in the sequel that the solution to this problem both in terms of displacements and stresses is unique, thus precluding instability and bifurcation phenomena. This notably requires that the free energy density $\tilde{\psi}$ of the solid is a polyconvex function of the local deformation gradient $\underline{\underline{F}}^{s}$ (Ball, 1977; Ciarlet, 1986).

As far as the fluid is concerned, the external boundary $\mathcal{F}_{t}^{\rho}$ of the fluid domain $d \Omega_{t}^{f}$ appears as the image of the external boundary $\mathcal{F}_{o}^{e}$ of the fluid domain $d \Omega_{0}^{f}$ by the homogeneous transformation $\underline{x}=\underline{\underline{F}} \cdot \underline{X}$, and not by the actual transformation of the fluid. As a matter of fact, the transformation of the fluid has not to be considered in the present approach which considers the porous medium as an open system. In fact, the fluid response is characterized by the fluid pressure $p$. More precisely, each value of the fluid pressure is associated with a solution of (12), (13) and (14) and to a certain fluid volume (resp. fluid mass) in the domain $d \Omega_{t}^{f}$ delimited by $\mathcal{F}_{t}^{e}$ and $\mathcal{S}_{t}^{i}$. According to the specified loading parameter $(J \phi$ or $m$ ), the appropriate fluid pressure $p$ corresponds to the fluid volume $J \phi\left|d \Omega_{o}\right|$ or to the fluid mass change $m\left|d \Omega_{o}\right|$. Let us denote by $\underline{U}=\underline{\underline{F}} \cdot \underline{F}^{-1} \cdot \underline{x}$ the velocity associated with the homogeneous transformation $\underline{x}=\underline{\underline{F}} \cdot \underline{X}$, which differs from the velocity of the fluid. Considering given rates of the loading parameters $\underline{\underline{F}}$ and $J \phi$, the expression of the porous space volume rate $\overline{d \Omega_{t}^{f}}$ is:

$$
\overline{d \Omega_{t}^{f}}=\dot{\overline{J \phi}}\left|d \Omega_{o}\right|=\int_{\mathcal{F}_{i}^{e}} \underline{n} \cdot \underline{U} d a-\int_{\mathcal{S}_{i}^{i}} \underline{\dot{\Phi}}^{s} \cdot \underline{n} d a
$$


where $\underline{n}$ denotes the outer unit normal to the fluid on $\mathcal{F}_{t}^{e}$ [first term of (15)] and to the solid on $\mathcal{S}_{t}^{i}$ [second term of (15)] in the deformed configuration.

Let us now consider the total free energy of the two constituents contained in $d \Omega_{t}$, i.e. $\Psi\left|d \Omega_{o}\right|$

$$
\Psi\left|d \Omega_{o}\right|=\int_{d \Omega_{o}^{s}} \tilde{\psi}\left(\underline{\underline{\tilde{F}^{s}}}\right) d V_{o}+\varphi_{m}\left(m+\rho_{o}^{f} \phi_{o}\right)\left|d \Omega_{o}\right|
$$

We have seen that the resolution of the boundary value problem defined by Eqs. (12)-(14) allows determination of the transformation of the solid $\underline{\Phi}^{s}$ and the fluid pressure $p$ as functions of the macroscopic loading parameters $\underline{\underline{F}}$ and $m$ or $J \phi$. The fluid free energy density $\varphi_{m}$ being a function of $p,(16)$ shows that $\Psi$ is a function of $\underline{\underline{F}}$ and $m$ or $J \phi$ so that

$$
\dot{\Psi}=\frac{\partial \Psi}{\partial \underline{\underline{F}}}(\underline{\underline{F}}, m):{ }^{T} \underline{\underline{\underline{F}}}+\frac{\partial \Psi}{\partial m}(\underline{\underline{F}}, m) \dot{m}=\frac{\partial \Psi}{\partial \underline{\underline{F}}}(\underline{\underline{F}}, J \phi):{ }^{T} \underline{\underline{\underline{F}}}+\frac{\partial \Psi}{\partial(J \phi)}(\underline{\underline{F}}, J \phi) \dot{\bar{J} \phi}
$$

We first examine the particular case of an incompressible fluid for which (6) will be derived. Thereafter, we shall see that (11) is the most convenient form of the state equations in the case of compressible fluids.

\subsection{Case of an incompressible fluid}

Each state equation in (6) can be associated with an experiment conducted on the representative elementary volume. The first one is an undrained experiment where $\underline{\underline{F}} \neq 0$ and $\dot{m}=0$, whereas the second one corresponds to a given fluid mass rate $\dot{m} \neq 0$ in an instantaneously fixed configuration of the skeleton $(\underline{\underline{F}}=0)$.

\subsubsection{Undrained evolution: $\dot{m}=0, \underline{\underline{\underline{F}}} \neq 0$}

In the case of an undrained experiment, the incompressibility of the fluid implies that the porous space volume rate $\overline{d \Omega_{t}^{f}}$ is equal to 0 . The kinematic relationship (15) then yields

$$
\dot{\overline{J \phi}}=0 \Longrightarrow \int_{\mathcal{F}_{i}^{e}} \underline{U} \cdot \underline{n} d a=\int_{\mathcal{S}_{i}^{i}} \underline{\dot{\Phi}}^{s} \cdot \underline{n} d a
$$

The free energy density $\varphi_{m n}$ being a constant in the case of an incompressible fluid, the differentiation of (16) with respect to time gives

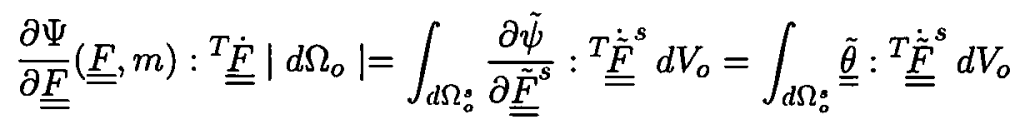

where (12) has been used. Let us denote by $\underline{\underline{d}}$ the strain rate tensor associated with the velocity field $\underline{\dot{\Phi}}^{s}$. We then have

$$
\tilde{J}^{s} \underline{\underline{\tilde{\sigma}}}: \underline{\underline{\tilde{d}}}=\underline{\underline{\tilde{\theta}}}:{ }^{T} \underline{\underline{\tilde{\tilde{F}}}}^{s}
$$

which can be introduced in (19)

$$
\dot{\Psi}\left|d \Omega_{o}\right|=\frac{\partial \Psi}{\partial \underline{\underline{F}}}(\underline{\underline{F}}, m):{ }^{T} \underline{\underline{\dot{F}}}\left|d \Omega_{o}\right|=\int_{d \Omega_{i}^{s}} \underline{\underline{\tilde{\sigma}}}: \underline{\underline{\tilde{d}}} d V=\int_{\mathcal{S}_{i}^{e}} \underline{\underline{U}} \cdot \underline{\underline{\tilde{\sigma}}} \cdot \underline{\underline{n}} d a+\int_{\mathcal{S}_{i}^{i}} \underline{\dot{\Phi}}^{s} \cdot \underline{\underline{\tilde{\sigma}}} \cdot \underline{n} d a
$$


The stress vector $\underline{\underline{\tilde{\sigma}}} \cdot \underline{n}$ on $\mathcal{S}_{t}^{i}$ is equal to $-p \underline{n}$. Besides, the Cauchy stress field $\underline{\underline{\tilde{g}}}$ is uniform in $d \Omega_{t}^{f}$, equal to $-p \underline{\underline{1}}$. Using (18), (21) can be transformed into

$$
\frac{\partial \Psi}{\partial \underline{\underline{F}}}(\underline{\underline{F}}, m):{ }^{T} \underline{\underline{\underline{F}}}\left|d \Omega_{o}\right|=\int_{\mathcal{S}_{t}^{e}} \underline{U} \cdot \underline{\underline{\tilde{\sigma}}} \cdot \underline{n} d a+\int_{\mathcal{F}_{i}^{e}} \underline{\underline{U}} \cdot \underline{\underline{\tilde{\sigma}}} \cdot \underline{n} d a=\left(\int_{d \Omega_{t}} \underline{\underline{\tilde{\sigma}}} d V\right): \underline{\underline{\underline{F}}} \cdot \underline{\underline{F}}^{-1}
$$

since $\underline{U}=\underline{\underline{\dot{F}}} \cdot \underline{\underline{F}}^{-1} \cdot \underline{x}$. Using the symmetry of the Cauchy stress tensor $\underline{\underline{\tilde{\sigma}}}$, it may then be concluded that

$$
J\left(\frac{1}{\left|d \Omega_{t}\right|} \int_{d \Omega_{t}} \underline{\underline{\tilde{\sigma}}} d V\right) \cdot{ }^{T} \underline{\underline{F}}^{-1}=\frac{\partial \Psi}{\partial \underline{\underline{F}}}(\underline{\underline{F}}, m)
$$

which provides an interpretation of the first state equation in (6) at the microscopic level. More precisely, the left hand side of (23) reveals that the macroscopic Boussinesq stress tensor $\underline{\underline{\theta}}$ is the Lagrangian stress tensor which corresponds to the mean value of the Cauchy stress field over $d \Omega_{t}$, through the transformation of the skeleton characterized by $\underline{\underline{F}}$.

Alternatively, it is natural to attempt to relate $\underline{\underline{\theta}}$ to the microscopic Boussinesq stress field $\underline{\tilde{\theta}}$ on $d \Omega_{o}$. In the solid domain, the Boussinesq stress tensor has already be defined by the solid constitutive Eq. (12). As far as the fluid is concerned, we have to deal with the fact that the actual transformation of the fluid is irrelevant to our approach. Thus, in order to define a Lagrangian stress tensor on the fluid domain $d \Omega_{o}^{f}$, we now introduce a virtual transformation of the fluid $\underline{x}=\underline{\Phi}^{f}(\underline{X})$, which is an extension to $d \Omega_{0}^{f}$ of the transformation $\underline{\Phi}^{s}$ already defined on the solid domain $d \Omega_{o}^{s}$. It is subjected to be equal to the transformation of the solid on $\mathcal{S}_{o}^{i}$ and to the homogeneous transformation $\underline{x}=\underline{\underline{F}} \cdot \underline{X}$ on $\mathcal{F}_{o}^{e}$. It follows that

$$
\begin{array}{ll}
\tilde{J}^{f T} \underline{\underline{\underline{F}}}^{f-1} \cdot \underline{N}=\tilde{J}^{s} \underline{\underline{F}}^{s}-1 \cdot \underline{N} \text { on } \mathcal{S}_{o}^{i} \\
\tilde{J}^{f T} \underline{\underline{\tilde{F}}}^{f-1} \cdot \underline{N}=J^{T} \underline{\underline{F}}^{-1} \cdot \underline{N} & \text { on } \mathcal{F}_{o}^{e}
\end{array}
$$

where $\underline{\underline{\tilde{F}}}^{f}=\underline{\underline{\nabla}}_{\mathrm{X}} \underline{\Phi}^{f}$ and $\tilde{J}^{f}=\operatorname{det} \underline{\underline{\tilde{F}}}^{f}$. Relations (24) and (25) simply express that any material surface element located on $\mathcal{S}_{o}^{i}$ and $\mathcal{F}_{o}^{e}$ is transformed in the same way through $\underline{\Phi}^{s}$ and $\underline{\Phi}^{f}$. Of course, such a virtual transformation of $d \Omega_{0}^{f}$ is non-unique. The Cauchy stress field in $d \Omega_{t}^{f}$ being equal to $-p \underline{\underline{1}}$, the Boussinesq stress field associated to a given extension $\underline{\Phi}^{f}$ and accordingly defined as $-p \tilde{J}^{f} \underline{\underline{F}}^{f}-1$ is also non-unique. However, using the following mathematical result

$$
\int_{\partial \Omega} \underline{\varphi} \cdot \underline{\underline{\tilde{\theta}}} \cdot \underline{N} d A+\int_{\Omega} \underline{\varphi} \cdot \operatorname{Div} \underline{\underline{\theta}} d V=\int_{\Omega} \underline{\underline{\tilde{\theta}}}:{ }^{T}\left(\underline{\underline{\nabla}}_{\lambda} \varphi\right) d V
$$

with $\Omega=d \Omega_{o}^{f}$ and $\underline{\varphi}=X_{j} \underline{e}_{i}$, and observing that $\operatorname{Div} \underline{\underline{\tilde{\theta}}}=0$, one obtains, using (24) and (25)

$$
\int_{d \Omega_{o}^{j}} \tilde{\theta}_{i j} d V_{o}=p \int_{\mathcal{S}_{o}^{i}} X_{j} \underline{e}_{i} \cdot \tilde{J}^{s} \underline{\underline{F}}^{s}-1 \underline{N} d A-p \int_{\mathcal{F}_{o}^{e}} X_{j} \underline{e}_{i} \cdot J^{T} \underline{\underline{F}}^{-1} \cdot \underline{N} d A
$$

This establishes that the mean value of $\underline{\underline{\tilde{\theta}}}$ over the fluid domain $d \Omega_{o}^{f}$ is independent of the particular choice made for the extension $\underline{\Phi}^{f}$ of $\underline{\Phi}^{s}$.

If we now apply (26) with $\Omega=d \Omega_{o}^{s}$ and $\underline{\varphi}=\underline{\Phi}^{s}$, (19) takes the form

$$
\frac{\partial \Psi}{\partial \underline{\underline{F}}}(\underline{\underline{F}}, m):{ }^{T} \underline{\underline{\underline{F}}}\left|d \Omega_{o}\right|=\int_{\mathcal{S}_{o}^{i}} \underline{\dot{\Phi}}^{s} \cdot \underline{\underline{\tilde{\theta}}} \cdot \underline{N} d A+\int_{\mathcal{S}_{o}}(\underline{\underline{\underline{F}}} \cdot \underline{X}) \cdot \underline{\underline{\theta}} \cdot \underline{N} d A
$$


Besides, using (13) and (25), one obtains the Lagrangian formulation of (18) in the form

$$
\int_{\mathcal{S}_{o}^{i}} \underline{\dot{\Phi}}^{s} \cdot \underline{\underline{\theta}} \cdot \underline{N} d A=-p \int_{\mathcal{F}_{o}}(\underline{\underline{F}} \cdot \underline{X}) \cdot J^{T} \underline{\underline{F}}^{-1} \cdot \underline{N} d A=\int_{\mathcal{F}_{o}}(\underline{\underline{\dot{F}}} \cdot \underline{X}) \cdot \underline{\underline{\theta}} \cdot \underline{N} d A
$$

which can be introduced in (28)

$$
\frac{\partial \Psi}{\partial \underline{\underline{F}}}(\underline{\underline{F}}, m):{ }^{T} \underline{\underline{\underline{F}}}\left|d \Omega_{0}\right|=\int_{\partial\left(d \Omega_{o}\right)}(\underline{\underline{\dot{F}}} \cdot \underline{X}) \cdot \underline{\underline{\tilde{\theta}}} \cdot \underline{N} d A=\left(\int_{d \Omega_{0}} \underline{\underline{\hat{\theta}}} d V_{o}\right):{ }^{T} \underline{\underline{\dot{F}}}
$$

where (26) has been used once again with $\Omega=d \Omega_{o}$ and $\underline{\varphi}=\underline{\underline{F}} \cdot \underline{X}$. The comparison of (23) and (30) shows that

$$
J\left(\frac{1}{\left|d \Omega_{t}\right|} \int_{d \Omega_{t}} \underline{\underline{\tilde{\sigma}}} d V\right) \cdot{ }^{T} \underline{\underline{F}}^{-1}=\frac{1}{\left|d \Omega_{t}\right|} \int_{d \Omega_{\mathrm{o}}} \underline{\underline{\tilde{\theta}}} d V_{o}=\frac{\partial \Psi}{\partial \underline{\underline{F}}}(\underline{\underline{F}}, m)
$$

which means that the macroscopic Boussinesq tensor associated with the mean Cauchy stress tensor over $d \Omega_{t}$ is also the average value of the Boussinesq stress field calculated over the whole elementary volume $d \Omega_{o}$.

3.1.2. Fluid mass exchange $(\dot{m} \neq 0)$ with instantaneously fixed configuration: $\underline{\underline{F}}=0$

Condition $\underline{\underline{F}}=0$ which implies that $\underline{U}=0$ in (15) combined with (1) and the fluid incompressibility yields:

$$
\underline{\underline{F}}=0 \Longrightarrow \dot{\bar{J} \phi}\left|d \Omega_{o}\right|=\frac{\dot{m}}{\rho^{f}}\left|d \Omega_{o}\right|=-\int_{\mathcal{S}_{i}^{i}} \dot{\Phi}^{s} \cdot \underline{n} d a
$$

Differentiating (16) with respect to time gives on account of (17)

$$
\dot{\Psi}\left|d \Omega_{o}\right|=\dot{m} \frac{\partial \Psi}{\partial m}(\underline{\underline{F}}, m)\left|d \Omega_{o}\right|=\dot{m} \int_{d \Omega_{o}^{s}} \underline{\underline{\tilde{\theta}}}: \frac{\partial^{T} \underline{\underline{\underline{F}}}^{s}}{\partial m} d V_{o}+\varphi_{m} \dot{m}\left|d \Omega_{o}\right|
$$

with

$$
\tilde{J}^{s} \underline{\underline{\tilde{\sigma}}}: \underline{\underline{\tilde{d}}}=\underline{\underline{\tilde{\theta}}}: \frac{\partial^{T} \underline{\underline{\underline{\underline{F}}}}^{s}}{\partial m} \dot{m}
$$

where once again $\underline{\underline{d}}$ denotes the strain rate tensor associated with the velocity field $\underline{\Phi}^{s}$. Using the boundary conditions (13) along with the condition $\underline{\underline{F}}=0$, it is readily seen that

$$
\int_{d \Omega_{i}^{s}} \underline{\underline{\tilde{\sigma}}}: \underline{\underline{d}} d V=\int_{\mathcal{S}_{i}^{i}} \underline{\dot{\Phi}}^{s} \cdot \underline{\underline{\tilde{\sigma}}} \cdot \underline{n} d a=-p \int_{\mathcal{S}_{i}^{i}} \underline{\dot{\Phi}}^{s} \cdot \underline{n} d a
$$

The introduction of (32), (34) and (35) in (33) finally yields

$$
\dot{m} \frac{\partial \Psi}{\partial m}(\underline{\underline{F}}, m)\left|d \Omega_{o}\right|=\int_{d \Omega_{i}^{s}} \underline{\underline{\tilde{\sigma}}}: \underline{\underline{d}} d V+\varphi_{m} \dot{m}\left|d \Omega_{o}\right|=\left(\frac{p}{\rho^{f}}+\varphi_{m}\right) \dot{m}\left|d \Omega_{o}\right|
$$

from which we derive the second state equation in (6)

$$
g_{m}=\frac{p}{\rho^{f}}+\varphi_{m}=\frac{\partial \Psi}{\partial m}(\underline{\underline{F}}, m)
$$




\subsection{Case of compressible fluid}

We now establish the state equations (11) by following a micro-macro reasoning of the same kind as that performed in the previous section. According to (7) and (16), the expression of $\Psi^{s}$ in terms of microscopic fields is

$$
\Psi^{s}\left|d \Omega_{o}\right|=\int_{d \Omega_{o}^{s}} \tilde{\psi}\left(\underline{\underline{\tilde{F}}}^{s}\right) d V_{o}
$$

Each state equation in (11) may again be associated with an experiment. The first one corresponds to a condition of instantaneously constant porous space volume, i.e. $\dot{d \Omega_{t}}$ or $\dot{\overline{J \phi}}=0$. Using (15), this condition is exactly equivalent to condition $\dot{m}=0$ in the case of an incompressible fluid, which was given in (18). Consequently, replacing $m$ by $J \phi$ and $\Psi$ by $\Psi^{s}$ in (19), (20), (21), (22) as well as in (28) and (30), one obtains

$$
J\left(\frac{1}{\left|d \Omega_{t}\right|} \int_{d \Omega_{t}} \underline{\underline{\tilde{\sigma}}} d V\right) \cdot \underline{T}^{T} \underline{\underline{F}}^{-1}=\int_{d \Omega_{0}} \underline{\underline{\tilde{\theta}}} d V_{o}=\frac{\partial \Psi^{s}}{\partial \underline{\underline{F}}}(\underline{\underline{F}}, J \phi)
$$

In the second experiment, the porous space volume rate $\dot{\overline{J \phi}}$ is prescribed in an instantaneously fixed configuration of the (macroscopic) skeleton particle $(\underline{\underline{\underline{F}}}=0)$ ). The flux of $\underline{\dot{\Phi}}^{s}$ through the boundary $\mathcal{S}_{t}^{i}$ is then related as in (32) to $\dot{\overline{J \phi}}$

$$
\dot{\underline{F}}=0 \rightarrow \dot{\overline{J \phi}}\left|d \Omega_{o}\right|=-\int_{\mathcal{S}_{i}^{i}} \dot{\Phi}^{s} \cdot \underline{n} d a
$$

Taking (17) into account, the differentiation of (38) with respect to time writes

$$
\dot{\bar{J} \Phi} \frac{\partial \Psi^{s}}{\partial(J \Phi)}(\underline{\underline{F}}, J \Phi)\left|d \Omega_{o}\right|=\dot{\bar{J} \Phi} \int_{d \Omega_{o}^{s}} \underline{\tilde{\theta}}: \frac{\partial^{T} \underline{\underline{\underline{F}}}^{s}}{\partial(J \Phi)} d V_{o}
$$

Using condition (40), it follows that

$$
\dot{\overline{J \Phi}} \int_{d \Omega_{o}^{s}} \underline{\underline{\tilde{\theta}}}: \frac{\partial^{T} \underline{\underline{\underline{F}}}^{s}}{\partial(J \Phi)} d V_{o}=\int_{d \Omega_{t}^{s}} \underline{\underline{\tilde{\sigma}}}: \underline{\underline{\tilde{d}}} d V=\int_{\mathcal{S}_{i}^{i}} \underline{\dot{\Phi}}^{s} \cdot \underline{\underline{\tilde{\sigma}}} \cdot \underline{n} d a=p \dot{\bar{J} \phi}\left|d \Omega_{o}\right|
$$

so that, combining (41) and (42) finally yields the second state equation of (11)

$$
p=\frac{\partial \Psi^{s}}{\partial(J \phi)}(\underline{\underline{F}}, J \phi)
$$

\section{Validity of the effective stress concept in finite strain poroelasticity}

As already mentioned, finite strain poroelasticity provides an appropriate framework for describing the behaviour of biological structures such as the heart muscle (Van Campen et al:, 1994) or human lungs (Kowalczyk and Kleiber, 1994). The role of the interstitial fluid in the mechanical response of the porous medium is simply taken into account by postulating that the deformations of the skeleton are governed by the so-called "effective stress" defined on the current configuration as

$$
\underline{\underline{\sigma}}^{\prime}=\underline{\underline{\sigma}}+p \underline{\underline{1}}
$$


More precisely, considering that $\underline{\underline{\sigma}}$ is a function of $\underline{\underline{F}}$ and $p$ taken as independent variables, the Terzaghi's effective stress principle writes

$$
\underline{\underline{\sigma}}(\underline{\underline{F}}, p)+p \underline{\underline{1}}=\underline{\underline{\sigma}}(\underline{\underline{F}}, 0)
$$

which can be transformed in terms of Boussinesq stress tensors (2) into

$$
\underline{\underline{\theta}}(\underline{\underline{F}}, p)+p J^{T} \underline{\underline{F}}^{-1}=\underline{\underline{\theta}}(\underline{\underline{F}}, 0)
$$

or, multiplying by ${ }^{T} \underline{\underline{\underline{F}}}$

$$
\underline{\underline{\theta}}(\underline{\underline{F}}, p):{ }^{T} \underline{\underline{\underline{F}}}+p \dot{J}=\underline{\underline{\theta}}(\underline{\underline{F}}, 0):{ }^{T} \underline{\underline{\underline{F}}}
$$

since it is to be noticed that

$$
\dot{J}=J \underline{\underline{\dot{F}}}: \underline{\underline{F}}^{-1}=J^{T} \underline{\underline{F}}^{-1}:{ }^{T} \underline{\underline{\dot{F}}}
$$

Now, (10) with $D=0$ gives

$$
\dot{\Psi}^{s}=\underline{\underline{\theta}}:{ }^{T} \underline{\underline{F}}+p \dot{\bar{J} \phi}
$$

Hence, on account of (47)

$$
\dot{\Psi}^{s}+p(\dot{J}-\dot{\overline{J \phi}})=\underline{\underline{\theta}}(\underline{\underline{F}}, 0):{ }^{T} \underline{\underline{\underline{F}}}
$$

Considering $\Psi^{s}$ and $J \phi$ as functions of the loading parameters $\underline{\underline{F}}$ and $p$ prescribed on the solid part at the microscopic scale, one obtains from (50)

$$
\left(\frac{\partial \Psi^{s}}{\partial \underline{\underline{F}}}+p\left(J^{T} \underline{\underline{F}}^{-1}-\frac{\partial J \phi}{\partial \underline{\underline{F}}}\right)\right):{ }^{T} \underline{\underline{\underline{F}}}+\left(\frac{\partial \Psi^{s}}{\partial p}-p \frac{\partial J \phi}{\partial p}\right) \dot{p}=\underline{\underline{\theta}}(\underline{\underline{F}}, 0):{ }^{T} \underline{\underline{\underline{F}}}
$$

which is valid whatever $T_{\underline{\underline{F}}}^{\underline{\underline{x}}}$ and $\dot{p}$, so that

$$
\begin{gathered}
\frac{\partial \Psi^{s}}{\partial \underline{\underline{F}}}+p\left(J^{T} \underline{\underline{F}}^{-1}-\frac{\partial J \phi}{\partial \underline{\underline{F}}}\right)=\underline{\underline{\theta}}(\underline{\underline{F}}, 0) \\
\frac{\partial \Psi^{s}}{\partial p}-p \frac{\partial J \phi}{\partial p}=0
\end{gathered}
$$

Differentiating (52) and (53) with respect to $p$ and $\underline{\underline{F}}$ respectively yields

$$
\frac{\partial J \phi}{\partial \underline{\underline{F}}}=J^{T} \underline{\underline{F}}^{-1}=\frac{\partial J}{\partial \underline{\underline{F}}}
$$

which means that $J(1-\phi)$ is a function of the pore pressure $p$ only

$$
J(1-\phi)=f(p)
$$


It follows from (53) and (55) that

$$
\frac{\partial \Psi^{s}}{\partial p}=-p f^{\prime}(p)
$$

that is

$$
\Psi^{s}(\underline{\underline{F}}, p)=\alpha(\underline{\underline{F}})+\beta(p)
$$

Several remarks should be made concerning this additive decomposition of the total free energy stored in the solid when subjected to $\underline{\underline{F}}$ and $p$.

a) It can be first observed that (55) and (57) prove to be necessary and sufficient conditions for obtaining an effective stress formulation. Indeed, $p$ being regarded as a function of $\underline{\underline{F}}$ and $J \phi$, the total free energy of the solid writes as follows

$$
\Psi^{s}(\underline{\underline{F}}, J \phi)=\alpha(\underline{\underline{F}})+\gamma(J-J \phi)
$$

Thus, the constitutive equations (11) take the form

$$
\underline{\underline{\theta}}=\frac{\partial \Psi^{s}}{\partial \underline{\underline{F}}}(\underline{\underline{F}}, J \phi)=\frac{\partial \alpha}{\partial \underline{\underline{F}}}+\gamma^{\prime} \frac{\partial J}{\partial \underline{\underline{F}}}, p=\frac{\partial \Psi^{s}}{\partial(J \phi)}(\underline{\underline{F}}, J \phi)=-\gamma^{\prime}
$$

or, by eliminating $\gamma^{\prime}$

$$
\underline{\underline{\theta}}=-p \frac{\partial J}{\partial \underline{\underline{F}}}=\underline{\underline{\theta}}(\underline{\underline{F}}, p)+J p^{T} \underline{\underline{F}}^{-1}=\frac{\partial \alpha}{\partial \underline{\underline{F}}}
$$

which is equivalent to (46) since $\frac{\partial \alpha}{\partial \underline{F}}$ is independent of $p$.

b) These conditions are automatically fulfilled in the case of an incompressible solid matrix. In such a case, the volume of the solid domain $\left|d \Omega_{t}^{s}\right|=J(1-\phi)\left|d \Omega_{o}\right|$ remains constant, equal to $\left(1-\phi_{o}\right)\left|d \Omega_{o}\right|$, so that $f(p)=1-\phi_{o}$ in $(55)$ and $\Psi^{s}(\underline{F}, p)$ depends on $\underline{\underline{F}}$ only. Such a result may be directly obtained by referring to the solutions of the boundary value problems attached to the solid domain corresponding to $(\underline{\underline{F}}, p=0)$ and $(\underline{\underline{F}}, p)$ respectively.

Let $\underline{\underline{x}}=\underline{\Phi}_{o}^{s}(\underline{X})$ be the function associated with the solution to this problem for $(\underline{\underline{F}}, p=0)$. The corresponding stress solution is

$$
\begin{array}{r}
\underline{\underline{\sigma}}_{o}=\frac{1}{\tilde{J}_{o}^{s}} \frac{\partial \tilde{\psi}}{\partial \underline{\underline{\tilde{F}}}_{o}^{s}} \cdot{ }^{T} \underline{\underline{\tilde{F}}}_{o}^{s}+\tilde{\eta}_{o} \underline{\underline{1}} \text { in } d \Omega_{t}^{s} \\
\underline{\underline{\sigma}}_{o}=0 \text { in } d \Omega_{t}^{f}
\end{array}
$$

where $\underline{\underline{\tilde{F}}}_{o}^{s}=\underline{\underline{\nabla}}_{X} \Phi_{o}^{s}$, with $\tilde{J}_{o}^{s}=\operatorname{det} \underline{\underline{F}}_{o}^{s}=1$, and $\tilde{\eta}_{o}$ is a scalar field, the local value of which at any point of the solid representing the Lagrange multiplier associated with the incompressibility constraint. The stress field $\underline{\underline{\sigma}}_{0}$ must comply with the equilibrium conditions

$$
\operatorname{div} \underline{\underline{\tilde{\sigma}}}_{0}=0 \text { in } d \Omega_{t}^{s} ; \underline{\tilde{\sigma}}_{0} \cdot \underline{n}=0 \text { on } \mathcal{S}_{t}^{i}
$$


Likewise, let $\left(\underline{\Phi}_{p}^{s}, \underline{\tilde{\sigma}}_{l}\right)$ be the solution corresponding to $(\underline{\underline{F}}, p)$. The stress field $\underline{\tilde{\sigma}}_{p}$, must be of the form

$$
\begin{array}{r}
\underline{\underline{\sigma}}_{p}=\frac{1}{\tilde{J}^{s}} \frac{\partial \tilde{\psi}}{\partial \underline{\underline{\tilde{F}}}^{s}} \cdot{ }^{T} \underline{\underline{F}}^{s}+\tilde{\eta}_{p} \underline{\underline{1}} \text { in } d \Omega_{t}^{s} \\
\underline{\underline{\sigma}}_{p}=-p \underline{\underline{1}} \text { in } d \Omega_{t}^{f}
\end{array}
$$

and satisfy the equilibrium conditions

$$
\operatorname{div} \underline{\underline{\sigma}}_{1},=0 \text { in } d \Omega_{t}^{s} ; \underline{\tilde{\sigma}}_{1}, \underline{n}=-p \underline{n} \text { on } \mathcal{S}_{t}^{i}
$$

It can be easily shown that $\left(\underline{\Phi}_{p}^{s}, \underline{\tilde{\sigma}}_{p}\right)$ is such that

$$
\underline{\tilde{\Phi}}_{o}^{*}=\underline{\Phi}_{l}^{*} ; \underline{\tilde{\sigma}}_{1}=\underline{\underline{\sigma}}_{0}-p \underline{\underline{1}}
$$

which means that, the displacements being prescribed on $\mathcal{S}_{o}^{e}$ through $\underline{\underline{F}}$, the displacement solution is insensitive to the pressure $p$ applied on $\mathcal{S}_{t}^{i}$. Calculating the average values of both $\underline{\underline{\sigma}}_{0}$ and $\underline{\underline{\sigma}}_{l}$, over the whole representative volume $d \Omega_{t}$, it thus comes out from (67)

$$
\underline{\underline{\sigma}}(\underline{\underline{F}}, p)=\frac{1}{\left|d \Omega_{t}\right|} \int_{d \Omega_{t}} \underline{\tilde{\sigma}}_{l} d V=\frac{1}{\left|d \Omega_{t}\right|} \int_{d \Omega_{1}}\left(\underline{\underline{\sigma}}_{o}-p \underline{\underline{1}}\right) d V=\underline{\underline{\sigma}}(\underline{\underline{F}}, 0)-p \underline{\underline{1}}
$$

which establishes the validity of the effective stress principle.

\section{Conclusion}

Assuming that the solid matrix making up the skeleton phase of the porous medium at the microscopic scale behaves elastically, a micro-macro procedure has made it possible to derive the constitutive equations of finite strain poroelasticity. It relies upon a microscopic description of the saturated porous medium as a solid continuous phase containing a fluid-filled porous network and the related concept of representative volume. The link between the microscopic and macroscopic scales is established from this representative volume by imposing mixed boundary conditions on the solid domain attached to it. It thus appears in particular that, irrespective of the fluid compressibility, the two relevant variables are the deformation gradient $\underline{\underline{F}}$ of the skeleton and the porous space volume characterized by $J \phi$. The two force variables associated through the differentiation of the free energy of the solid $\Psi^{s}$ are the macroscopic Boussinesq stress tensor $\underline{\underline{\theta}}$, which has been proved to be equal to the mean value of the corresponding microscopic stress field calculated over the whole representative volume (including the fluid phase), and the pore pressure $p$.

As regards the possibility of formulating the poroelastic behaviour in terms of "effective stress" according to the so-called Terzaghi's principle, the micro-macro approach provides necessary and sufficient conditions for the validity of such a formulation: namely, that the volume of the solid domain is only pressure dependent and that its free energy may be additively decomposed into two terms depending on $\underline{F}$ and $p$ separately. It has been proved that such conditions are satisfied in the case of an incompressible solid matrix. It is more than likely that the incompressibility of the solid matrix also constitutes a necessary condition (even though the proof still needs to be supplied), since one can hardly imagine other situations where the above-mentioned conditions ensuring an effective stress formulation would be satisfied. 


\section{References}

Auriault J.-L., Sanchez-Palencia E., 1977, Etude du comportement macroscopique d'un milieu poreux saturé déformable, J. Méc., 16, $\mathrm{n}^{\circ} 4,575-603$.

Ball J. M., 1977, Convexity conditions and existence theorems in non-linear elasticity, Arch. Ration. Mech. Anal., 63, $337-403$.

Biot M. A., 1972, Theory of finite deformations of porous solids. Indiana Univ. Math. J., 21, 7, 597-620.

Biot M. A., 1977, Variational lagrangian-thermodynamics of non isothermal finite strain mechanics of porous solids and thermomolecular diffusion. Int. J. Solids Structures, 13, 579-597.

Cheng A. H. D., 1997, Material coefficients of anisotropic poroelasticity. Int. J. Rock Mech. Min. Sci., 34, $\mathrm{n}^{\circ} 2,199-205$.

Ciarlet P. G., 1986, Elasticité tridimensionnelle. Masson, Paris.

Cieszko M., Kubik J., 1996, Constitutive relations and internal equilibrium condition for fluid-saturated porous solids-non linear theory. Arch. Mech., 48, 5, 893-910.

Coussy O., 1989, Thermodynamics of saturated porous solids in finite deformation. Eur. J. Mech. A/Solids, 8, 1-14.

Coussy O. 1995, Mechanics of Porous Continua, John Wiley, N.Y.

Dangla P., Coussy O., 1996, Drainage and drying of deformable porous materials: ID case study. Proc. IUTAM Symp., Mechanics of Granular and Porous Materials.

Dormieux L., Stolz C., 1992, Variational approach for poroelastic medium. C. R. Acad. Sci. Paris, 315, série II, 407-412 (in French, abridged English version).

Hill R., 1971, On constitutive macro-variables for heterogeneous solids at finite strain. Proc. R. Soc. A, 326, 131-147.

Kowalczyk P., Kleiber M., 1994, Modelling and numerical analysis of stresses and strains in the human lung including tissues-gas interaction, Eur. J. Mech. A/Solids, 13, $\mathrm{n}^{\circ}$ 3, 367-393.

Ogden R.W., 1974, On the overall moduli of non-linear elastic composite materials. J. Mech. Phys. Solids, 22, $541-553$.

Thompson M., Willis J.R., 1991, A reformulation of the equations of anisotropic poroelasticity, J. Appl. Mech. ASME 58, $612-616$.

Van Campen D.H., Huyghe J.M., Bovendeerd P.H.M., Arts T., 1994, Biomechanics of the heart muscle. Eur. J. Mech. A/Solids, 13, special issue, 19-41. 